\title{
MEASUREMENTS OF NON-LEPTONIC WEAK DECAYS OF LAMBDA HYPERNUCLEI
}

\author{
Reinhard A. Schumacher \\ Department of Physics, Carnegie Mellon University, Pittsburgh, PA 15213
}

\begin{abstract}
Aspects of recent measurements of the mesonic and non-mesonic partial decay rates of ${ }_{\Lambda}^{5} \mathrm{He}$, and ${ }_{\Lambda}^{12} \mathrm{C}$ are discussed. We comment on what the experiments tell us about the spin-isospin structure of $\Delta S=1$ weak interaction in the nuclear environment.

\section{INTRODUCTION}

Hypernuclei can decay via the mesonic weak decay modes $\Lambda \rightarrow n+\pi^{0}$ and $\Lambda$ $\rightarrow p+\pi^{-}$or the nonmesonic weak decay modes $\Lambda+p \rightarrow n+p$ and $\Lambda+n \rightarrow n+n$. The total decay rate is the sum of the four partial rates for the respective processes: $\Gamma_{\text {Total }}=1 / \tau \equiv \Gamma_{\pi^{-}}+\Gamma_{\pi^{0}}+\Gamma_{\mathrm{p}}+\Gamma_{\mathrm{n}}$, where $\tau$ is the lifetime of the hypernuclear state. The description of these processes requires an effective weak Hamiltonian which takes into account modification of the weak force due to the presence of strongly interacting particles. The lowest order weak Hamiltonian for quark-quark interactions leads to transitions of both $\Delta I=1 / 2$ and $\Delta I=3 / 2$ with comparable strength, but empirically both kaon decay and free hyperon decay data suggest that the $\Delta I=1 / 2$ amplitude is about a factor of twenty stronger than the $\Delta \mathrm{I}=3 / 2$ amplitude. Measurements of the partial decay rates of hypernuclear systems aim to test and understand the structure of this interaction in nuclei.
\end{abstract}

\section{MESONIC DECAYS OF ${ }_{\Lambda}^{12} \mathrm{C}$}

Szymanski et $a l^{1]}$ have recently published measurements of the partial and total decay rates for ${ }_{\Lambda}^{5} \mathrm{He}$ and ${ }_{\Lambda}^{12} \mathrm{C}$. The experiments used the $\mathrm{A}\left(\mathrm{K}^{-}, \pi^{-}\right) \Lambda_{\Lambda} \mathrm{A}^{(*)}$ reaction to produce and tag hypernuclear states using a magnetic spectrometer. Total widths were obtained by measuring the time difference between formation and decay. Partial rates were obtained by detection of charged decay products in coincidence with the formation of hypernuclear states. For ${ }_{\Lambda}^{12} \mathrm{C}$, a recent $\pi^{0}$ partial rate value from Sakaguchi et al ${ }^{2]}$ was the first to directly detect $\pi^{0}$ s using NaI counters to detect the decay photons. Table 1 summarizes data for the ground state decay of ${ }_{\Lambda}^{12} \mathrm{C}$. The ratio $\Gamma_{\pi} / \Gamma_{\pi_{0}}$ determined from the two experiments is seen to be $<0.74$ (95\% C.L.). Note that the $\Delta \mathrm{I}=1 / 2$ rule predicts 2 for the free mesonic decay ratio, but calculations of Motoba et $^{3} \mathrm{l}^{3]}$ yield a value of $0.79 \pm .05$ in a model which includes Pauli blocking and optical model distortion of the pion wavefunction. Thus the data and theory are not inconsistent. Sakaguchi et al used the central value for Szymanski et al's $\Gamma_{\pi \text {-result in noting that }}$ $\Gamma_{\pi-} / \Gamma_{\pi_{0}}$ was much smaller than the theoretical number. The upper limit for $\Gamma_{\pi-}$ shown in Table 1 and in Ref (1) results from a careful evaluation of possible backgrounds contributing to the ground state region of the ${ }_{\Lambda}^{12} \mathrm{C}$ excitation spectrum. Because the mesonic decay modes are strongly Pauli suppressed in all but the lightest

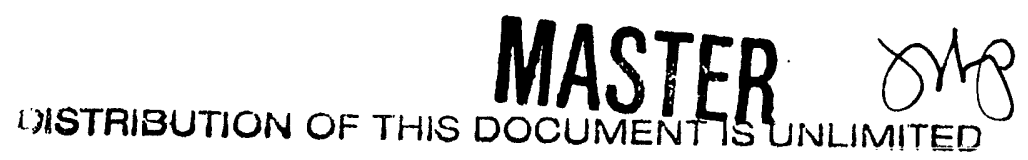




\section{DISCLAIMER}

This report was prepared as an account of work sponsored by an agency of the United States Government. Neither the United States Government nor any agency thereof, nor any of their employees, makes any warranty, express or implied, or assumes any legal liability or responsibility for the accuracy, completeness, or usefulness of any information, apparatus, product, or process disclosed, or represents that its use would not infringe privately owned rights. Reference herein to any specific commercial product, process, or service by trade name, trademark, manufacturer, or otherwise does not necessarily constitute or imply its endorsement, recommendation, or favoring by the United States Government or any agency thereof. The views and opinions of authors expressed herein do not necessarily state or reflect those of the United States Government or any agency thereof. 
nuclei, for carbon there is no distinct ground state peak when looking for decay pions in coincidence.

Table $1{ }_{\Lambda}^{12} \mathrm{C}$ ground state partial decay rates as a fraction of the free $\Lambda$ decay rate. The total non-mesonic rate is $\Gamma_{\mathrm{n} \cdot \mathrm{m} .} \equiv \Gamma_{\mathrm{p}}+\Gamma_{\mathrm{n}}$.

$$
\begin{array}{ccccl}
\Gamma_{\text {TOTAL }} / \Gamma_{\Lambda} & \Gamma_{\text {n.m. }} / \Gamma_{\Lambda} & \Gamma_{\pi-} / \Gamma_{\text {n.m. }} & \Gamma_{\pi \mathrm{o}} / \Gamma_{\Lambda} & \Gamma_{\pi-} / \Gamma_{\pi \mathrm{o}} \\
1.25 \pm 0.18 & 1.14 \pm 0.20 & <0.14(95 \% \text { C.L. }) & .217 \pm .074 & <0.74
\end{array}
$$

NON-MESONIC DECAYS OF ${ }_{\Lambda}^{4} \mathrm{He}$ AND ${ }_{\Lambda}^{5} \mathrm{He}$

The non-mesonic decay rates for $\Lambda+p \rightarrow n+p$ and $\Lambda+n \rightarrow n+n$ are sensitive to the spin-isospin structure of the strangeness changing weak interaction in nuclei. The recent status of the data and theory has been extensively reviewed by Cohen ${ }^{4]}$. Figure 1 illustrates the paths by which a $\Lambda \mathrm{N}$ initial state can decay non-mesonically to an $\mathrm{NN}$ final state. The $\Lambda \mathrm{n} \rightarrow \mathrm{nn}$ transition is forbidden by the Pauli principle for $\mathrm{I}=0$ final states. Note that comparing information obtained from several light hypernuclei, such as ${ }_{\Lambda}^{5} \mathrm{He}$ and ${ }_{\Lambda}^{4} \mathrm{He}$, can provide a way of extracting the strength of specific transitions. In lowest-order calculations involving one-pion exchange only, the ${ }^{3} \mathrm{~S}_{1} \rightarrow{ }^{3} \mathrm{D}_{1}$ transition dominates. The effects of initial state correlations and the inclusion of $\rho, K$, and other meson exchanges redistribute the decay strength to other spin channels, as shown in Figure 1 for the case of a calculation by Dubach ${ }^{5]}$. Model uncertainties in how to properly include meson exchanges beyond the pion have been emphasized by McKellar and Gibson ${ }^{6]}$, for example.

At a purely phenomenological level one can estimate directly from the data whether the $\Delta I=1 / 2$ Rule is satisfied by the data for non-mesonic weak decay. The rule requires, for any reaction mechanism, that the neutron stimulated rate is twice the proton stimulated rate for $I=1$ final states ${ }^{4]}$. Defining $R_{N S}$ as the rate for $\Lambda N \rightarrow N N$ from spin state $S$, it follows that $R_{n 0}=2 R_{p 0}$ and $R_{n 1} \leq 2 R_{p 1}$. What is measured experimentally are combinations of these quantities. One such combination is the neutron-toproton stimulated rate ratio for ${ }_{\Lambda}^{5} \mathrm{He}$,

$$
\gamma_{5} \equiv \frac{\Gamma_{\mathrm{n}}}{\Gamma_{\mathrm{p}}}\left({ }_{\Lambda}^{5} \mathrm{He}\right)=\frac{3 \mathrm{R}_{\mathrm{n} 1}+\mathrm{R}_{\mathrm{n} 0}}{3 \mathrm{R}_{\mathrm{p} 1}+\mathrm{R}_{\mathrm{p} 0}}=\frac{0.20 \pm 0.11}{0.21 \pm 0.07}=0.93 \pm 0.55,
$$

where the numerical values come from the recently published data of Szymanski et al ${ }^{1]}$. The $\Gamma_{\mathrm{n}}$ rate (in units of $\Gamma_{\Lambda}$ ) was revised prior to publication to reflect a more complete error analysis and better Monte Carlo correction of background due to $\pi^{-}$-induced neutrons. In the approach introduced by Block and Dalitz ${ }^{7]}$, and recently updated by Dover ${ }^{8]}$, one can further define

and

$$
\gamma_{4} \equiv \frac{\Gamma_{\mathrm{n}}}{\Gamma_{\mathrm{p}}}\left({ }_{\Lambda}^{4} \mathrm{He}\right)=\frac{2 \mathrm{R}_{\mathrm{n} 0}}{3 \mathrm{R}_{\mathrm{p} 1}+\mathrm{R}_{\mathrm{p} 0}}=0.45 \pm 0.17
$$




$$
\gamma \equiv \frac{\Gamma_{\mathrm{n} \cdot \mathrm{m} \cdot\left({ }_{\Lambda}^{4} \mathrm{He}\right)}}{\Gamma_{\mathrm{n} \cdot \mathrm{m} .}\left({ }_{\Lambda}^{4} \mathrm{H}\right)}=\frac{3 \mathrm{R}_{\mathrm{pl}}+\mathrm{R}_{\mathrm{p} 0}+2 \mathrm{R}_{\mathrm{n} 0}}{3 \mathrm{R}_{\mathrm{n} 1}+\mathrm{R}_{\mathrm{n} 0}+2 \mathrm{R}_{\mathrm{p} 0}}=0.7 \pm 0.15 .
$$

The numerical value for $\mathrm{Eq}(2)$ comes from $\mathrm{Ref} 9$ ], while the value for $\mathrm{Eq}(3)$ is an indirect estimate from several other measured numbers ${ }^{8]}$. Solving the three equations one obtains the ratios:

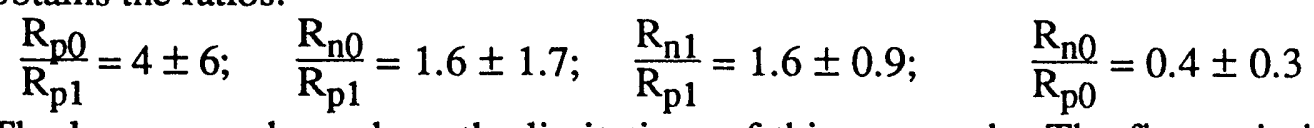

The large error bars show the limitations of this approach. The first ratio in Eq. (4) may suggest a large spin singlet component in non-mesonic decay, which is not predicted in typical models. The fourth ratio in Eq.(4) tests the $\Delta[=1 / 2$ Rule, which predicts this ratio to be 2 , suggesting a rather large violation in the case of light hypernuclei. Better data are clearly desirable to improve this analysis.

Pion exchange calculations ${ }^{5], 10]}$ of the nonmesonic rates agree with experiment in ${ }_{\Lambda}^{5} \mathrm{He}$ and ${ }_{\Lambda}^{12} \mathrm{C}$, as does a hybrid model calculation of the ${ }_{\Lambda}^{12} \mathrm{C}$ nonmesonic rate which uses quark-quark weak dynamics for small $\Lambda-\mathrm{N}$ separations and pion exchange for larger separations ${ }^{11]}$. Data for $\Gamma_{\mathrm{n}} / \Gamma_{\mathrm{p}}$ tend to be larger than theory and difficult to explain. One recent calculation ${ }^{12]}$ included the effects of $P$-shell configurations in the $\Lambda \mathrm{N}$ initial state, and concluded that the non-mesonic decays are difficult to understand in the context of any meson exchange model. All meson calculations done to date enforce the $\Delta \mathrm{I}=1 / 2$ rule by hand. Attempts to understand its origin in terms of quark dynamics have been only partially successful ${ }^{13]}$.

\section{NEW MEASUREMENTS ON ${ }_{\Lambda}^{4} \mathrm{He}$ AND ${ }_{\Lambda}^{5} \mathrm{He}$}

Data taking is complete for new determinations ${ }^{14]}$ of the mesonic and nonmesonic decay rates for ${ }_{\Lambda}^{4} \mathrm{He}$ and ${ }_{\Lambda}^{5} \mathrm{He}$. The principal improvement over existing data will come in the ${ }_{\Lambda}^{4} \mathrm{He}$ case, where a cryogenic target was used with carefully constructed "internal" veto scintillators for rejecting events in which $\pi^{-}$particles interact near the target to give spurious neutrons. This will allow a determination of $\Gamma_{\mathrm{n}}$, which is expected to be very small for ${ }_{\Lambda}^{4} \mathrm{He}$ but substantial for $\left.{ }_{\Lambda}^{5} \mathrm{He}(\operatorname{Ref} 5]\right)$. All four of the mesonic and non-mesonic rates will be extracted for these data, which form the thesis project for Michael Athanas of Carnegie Mellon.

\section{CONCLUSIONS}

The mesonic and non-mesonic decays of light hypernuclei are uniquely suited for studying the effective weak Hamiltonian of strangeness changing weak interactions. A simple phenomenological approach can be used to test the assumption that the $\Delta I=1 / 2$ Rule applies to non-mesonic decays inside the nucleus. Hypernuclear weak decay measurements are the most practical way to obtain data which can be related to the effective weak YN Hamiltonian. New measurements for light hypernuclei are currently being analyzed to obtain partial decay rates for all decay modes. 


\section{REFERENCES}

1) J.J.Szymanski et al., Phys. Rev. C 43, 849 (1991).

2) A. Sakaguchi et al, Phys. Rev. C 43, 73 (1991).

3) T. Motoba, K. Itonaga, H. Bando, Nucl. Phys. A489, 683 (1988).

4) Joseph Cohen, Progress in Particle and Nuclear Physics 25, 139 (1990).

5) John F. Dubach, Nuclear Physics A450, 71c (1986).

6) B.H.J.McKellar and B.F.Gibson, Phys. Rev. C 30, 322 (1984).

7) M.M. Block and R.H. Dalitz, Phys. Rev. Lett. 11, 96 (1963).

8) Carl B. Dover, Few Body Systems, Suppl. 2, 77 (1987).

9) M.M. Block et al, cited in Ref 8].

10) E. Oset and L.L.Salcedo, Nucl. Phys. A443, 704 (1985).

11) D. P. Heddle and L.S. Kisslinger, Phys. Rev. C 33, 608 (1986).

12) A. Ramos, C. Bennhold, E. van Meijgaard, and B.K.Jennings, preprint TRI-PP-91-12.

13) Frederick J. Gilman and Mark B. Wise, Phys. Rev. D 20, 2392 (1979).

14) Brookhaven AGS Experiment E788, G. Franklin and P.D.Barnes, spokesmen.

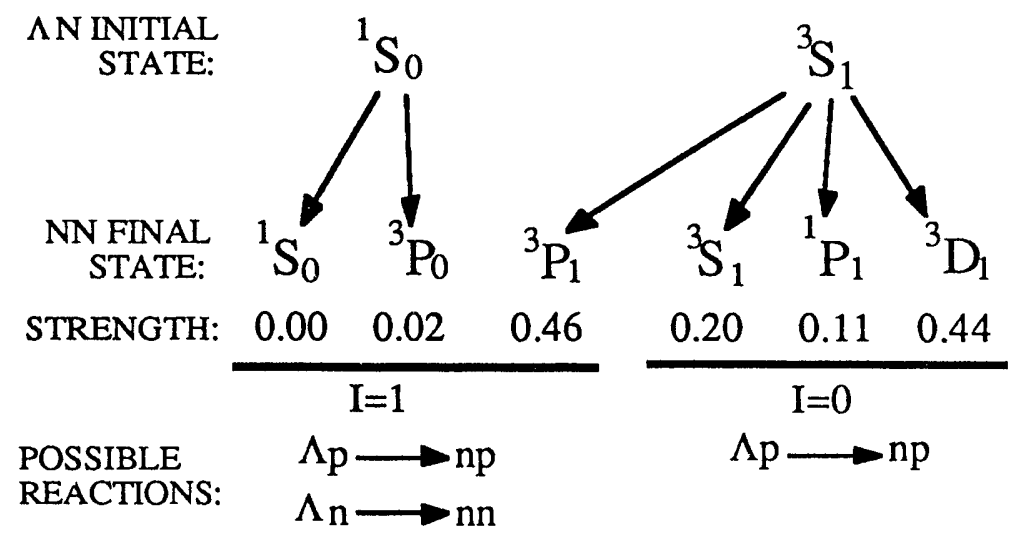

ALLOWED INITIAL STATES:

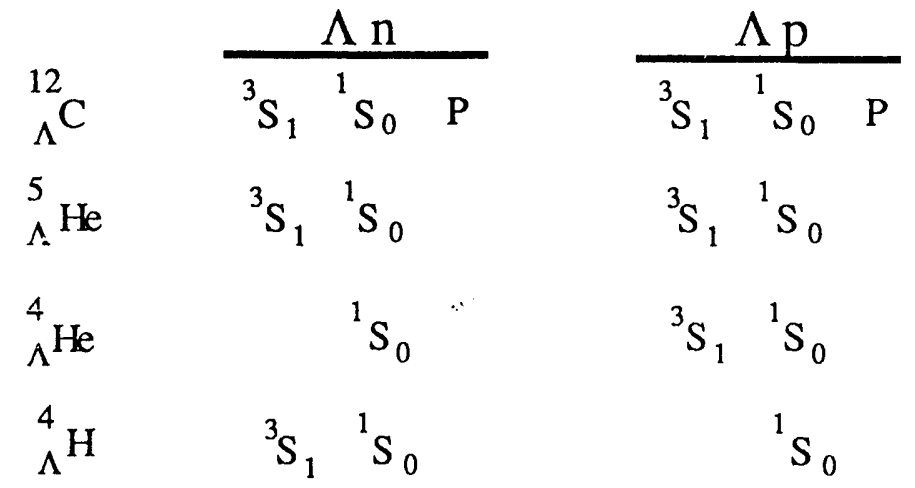

Figure 1) Spin and isospin combinations for non-mesonic weak decay of light hypernuclei. The predicted strengths for the separate channels are from Ref 5]. 

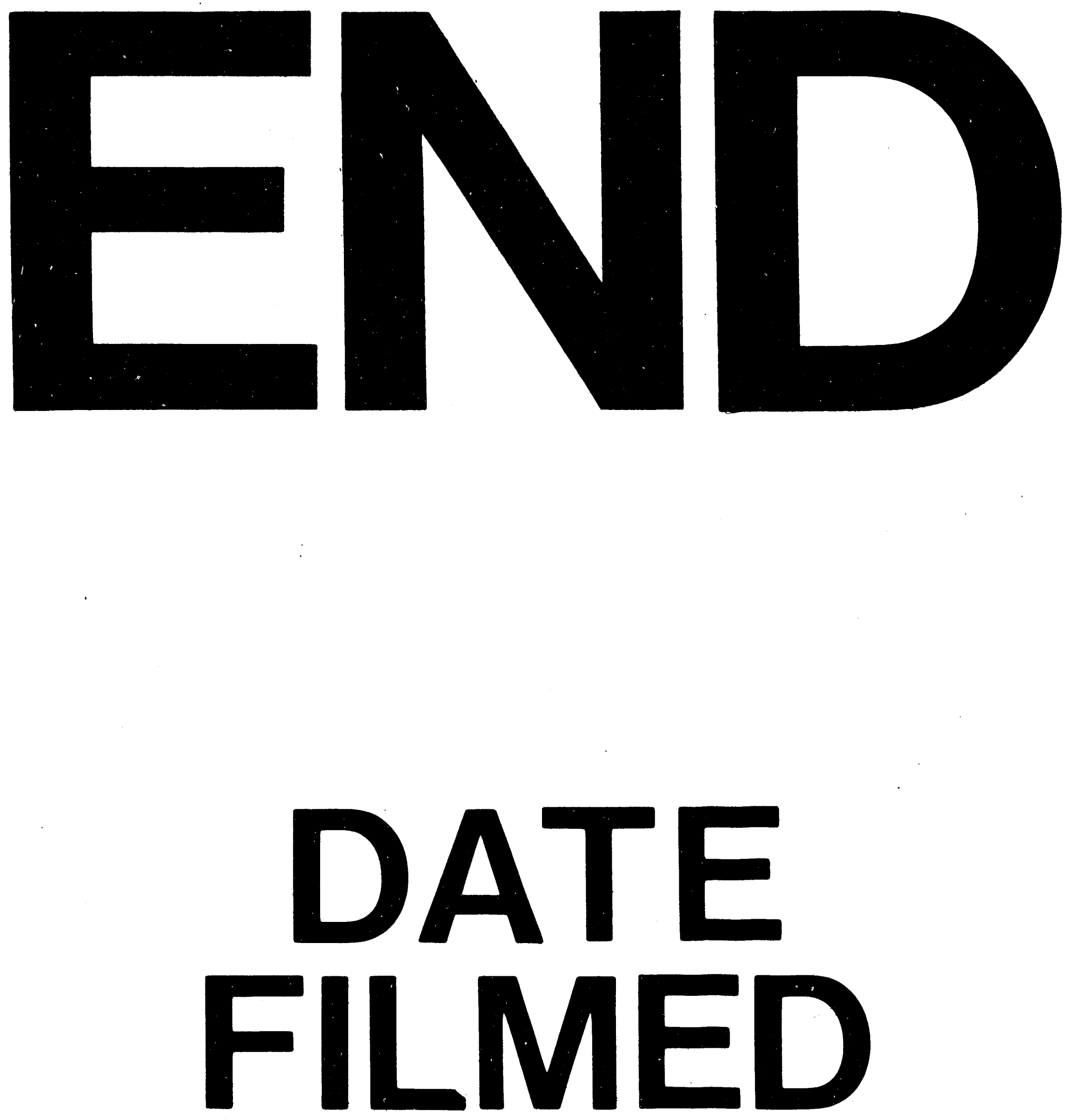

1

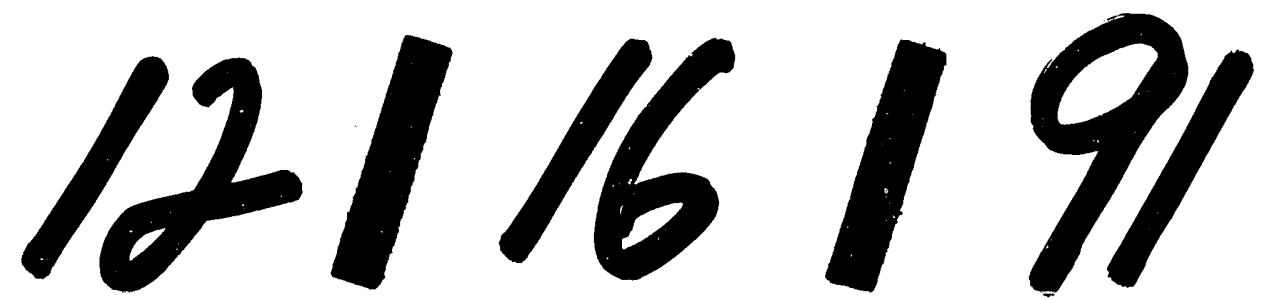


